\title{
Procedural sedation with dexmedetomidine for pediatric endoscopic retrograde cholangiopancreatography guided stone retraction
}

\author{
Byung Ju Ko, Jung-Hoon Jang, Jae Won Park, Seung Cheol Lee, and So Ron Choi
}

Department of Anesthesiology and Pain Medicine, Dong-A University Hospital, Busan, Korea

Pediatric endoscopic retrograde cholangiopancreatography (ERCP) is currently used as a diagnostic and therapeutic procedure [1]. Compared to adults, pediatric patients experience different physiological effects with respect to discomfort, anxiety, and pain responses. Therefore, appropriate analgesia and amnesia are more frequently required for pediatric patients. Various methods of sedation, including general anesthesia, can be used for pediatric ERCP [2].

Although the use of dexmedetomidine for sedation in pediatric patients in various circumstances is reported, few studies have reported its use in pediatric ERCP. Here, we report our experience with dexmedetomidine for ERCP-guided stone retraction in a pediatric patient.

A 10-year-old boy weighing $29 \mathrm{~kg}$ was referred to our department for deep sedation. He presented with obstructive jaundice, and a CT scan revealed a small stone at the distal common bile duct. Five days earlier, ERCP-guided stone retraction was performed under intravenous midazolam (3 doses of $0.1 \mathrm{mg}$ / $\mathrm{kg}$ ) sedation by a gastroenterologist. Stone retraction failed because of inadequate sedation, but the duct was successfully visualized using fluoroscopy.

ERCP-guided stone retraction was therefore rescheduled. Before the second procedure, informed consent was obtained from the patient's parents. After arrival at the ERCP suite, the patient was monitored using electrocardiography and pulse oximetry, and his noninvasive blood pressure (BP) and bispectral index (BIS) were checked. He was calm and cooperative. His vital signs, BIS values and Observer's Assess- ment of Alertness/Sedation scores were closely monitored and recorded (Table 1) during sedation.

The patient was positioned in the prone position with his head turned toward the operator. A $0.7 \mu \mathrm{g} / \mathrm{kg}$ loading dose of dexmedetomidine was intravenously administered over 10 minutes followed by a maintenance infusion of $0.5 \mu \mathrm{g} / \mathrm{kg} / \mathrm{h}$. Just prior to the procedure, $0.5 \mathrm{mg} / \mathrm{kg}$ ketamine and $0.5 \mu \mathrm{g} /$ $\mathrm{kg}$ fentanyl were intravenously administered consecutively. His oxygen saturation $\left(\mathrm{SpO}_{2}\right)$ decreased to $85 \%$ for a second. When the scope was inserted, his $\mathrm{SpO}_{2}$ recovered to $100 \%$. He breathed spontaneously with oxygen supplementation via nasal

Table 1. Values During the Procedure

\begin{tabular}{ccccccc}
\hline & $\begin{array}{c}\mathrm{SpO}_{2} \\
(\%)\end{array}$ & $\begin{array}{c}\text { SBP } \\
(\mathrm{mmHg})\end{array}$ & $\begin{array}{c}\mathrm{DBP} \\
(\mathrm{mmHg})\end{array}$ & $\begin{array}{c}\mathrm{HR} \\
(\mathrm{BPM})\end{array}$ & BIS & OAA/S score \\
\hline $\mathrm{T} 1$ & 98 & 126 & 78 & 75 & 98 & 4 \\
$\mathrm{~T} 2$ & 85 & 125 & 79 & 78 & 91 & 1 \\
$\mathrm{~T} 3$ & 100 & 128 & 76 & 76 & 90 & 1 \\
$\mathrm{~T} 4$ & 100 & 128 & 75 & 88 & 89 & 1 \\
$\mathrm{~T} 5$ & 100 & 105 & 54 & 72 & 78 & 1 \\
$\mathrm{~T} 6$ & 100 & 102 & 53 & 80 & 90 & 3 \\
T7 & 100 & 100 & 56 & 68 & 95 & 5 \\
\hline
\end{tabular}

Data are expressed as values. T1: start of dexmedetomidine infusion, T2: intravenous fentanyl and ketamine administration and start of the procedure, T3: 5 minutes after the start of the procedure, T4: 10 minutes after the start of the procedure, T5: end of the procedure, T6: 5 minutes after the end of procedure, T7: 10 minutes after the end of procedure. $\mathrm{SpO}_{2}$ : oxygen saturation, SBP: systolic blood pressure, DBP: diastolic blood pressure, HR: heart rate, BIS: bispectral index, OAA/S: Observer's Assessment of Alertness/Sedation.

Corresponding author: So Ron Choi, M.D., Ph.D., Department of Anesthesiology and Pain Medicine, Dong-A University Hospital, Dongdaeshin3-ga, Seo-gu, Busan 602-715, Korea. Tel: 82-51-240-5390, Fax: 82-51-247-7819, E-mail: choisr@dau.ac.kr

(c) This is an open-access article distributed under the terms of the Creative Commons Attribution Non-Commercial License (http:// creativecommons.org/licenses/by-nc/3.0/), which permits unrestricted non-commercial use, distribution, and reproduction in any medium, provided the original work is properly cited. 
prongs. The procedure was completed with minimal reductions in $\mathrm{BP}$ and heart rate.

After completing of the procedure, dexmedetomidine infusion was discontinued. The patient was then positioned in the supine position. He remained in the ERCP suite for 15 minutes, after which he was then discharged to the general ward, where his complete recovery was confirmed by anesthesiologists. The patient did not complain of postoperative nausea or vomiting and did not exhibit emergence agitation/delirium.

Dexmedetomidine is a highly selective $\alpha_{2}$ adrenoceptor agonist that has many advantages over other sedatives. In addition to producing sedative, analgesic, and anxiolytic effects, it provides respiratory stability [3]. It was first used as a sedative for patients being intubated in the intensive care unit. It was then used for sedation during many medical and surgical procedures without intubation. In addition, many studies have reported the use of dexmedetomidine in pediatric patients despite the lack of Food and Drug Administration approval [3]. Among pediatric patients, dexmedetomidine has been used for procedural sedation, particularly in diagnostic radiology.

As a sedative, dexmedetomidine maintains spontaneous respiration and preserves oxygenation and ventilation even at deep sedation. However, it is neither a complete anesthetic nor a complete analgesic [4]. Therefore, adjunctive analgesics are required during painful procedures.

Because the half-life of dexmedetomidine is relatively long, its use as a sedative is limited. Small doses and brief exposures may reduce the risk of prolonged recovery and delayed discharge from the recovery room and hospital [4].

In the present case, the total procedural and infusion times were short, and the patient recovered completely from the sedation within 30 minutes with no adverse events.
Sedation with topical pharyngeal anesthesia has developed into a safe and common alternative to general anesthesia in pediatric endoscopy because of short-acting opioids and specific narcotic and benzodiazepine medications. General anesthesia is still used in younger patients, particularly when intolerance in anticipated and after considering the higher complexity of procedures in patients with smaller anatomies [1].

In the present case, the stone was located in the distal common bile duct. Therefore, positional changes and other complicated situations were not required. Possible pain due to stone retraction was prevented with ketamine and fentanyl. Furthermore, visualization of the duct was previously performed during the first procedure; the stone removal was therefore fast and gentle. When patients are treated with appropriate analgesics, sedation with dexmedetomidine for pediatric ERCP would be safe and associated with better outcomes than sedation with other sedatives.

\section{References}

1. Paris C, Bejjani J, Beaunoyer M, Ouimet A. Endoscopic retrograde cholangiopancreatography is useful and safe in children. J Pediatr Surg 2010; 45: 938-42.

2. Wengrower D, Gozal D, Gozal Y, Meiri Ch, Golan I, Granot E, at al. Complicated endoscopic pediatric procedures using deep sedation and general anesthesia are safe in the endoscopy suite. Scand J Gastroenterol 2004; 39: 283-6.

3. Shukry M, Miller JA. Update on dexmedetomidine: use in nonintubated patients requiring sedation for surgical procedures. Ther Clin Risk Manag 2010: 6; 111-21.

4. Mason KP, Lerman J. Review article: Dexmedetomidine in children: current knowledge and future applications. Anesth Analg 2011; 113: 1129-42. 